and on gay hook-up apps and websites. Survey questions were analysed descriptively and included questions about the service itself, sexual health, technology use, and demographic characteristics.

Results Of 1272 participants completing the survey, 78\% identified as gay and $16 \%$ as bisexual, $73 \%$ identified as White, $52 \%$ reported being single, and 55\% reported living in the city of Vancouver. 32\% were aware of GCO, 13\% had visited the website and 3\% had tested through the service $(10 \%$ among the 411 men aware of GCO). Among GCO-aware participants, $50 \%$ intended to test through the service in the future (vs. 47\% among GCO-unaware), 51\% reported talking about GCO with others and 22\% knew someone who has used it. $46 \%$ reported that at times they would use GCO over their usual place of testing. The most common benefits reported by participants were testing without waiting for an appointment (50\%), getting results online (46\%), and saving time $(38 \%)$. The most common drawbacks were not speaking with a doctor or nurse (39\%), not being sure how the service works $(26 \%)$, and worrying about the privacy of one's online information (20\%).

Conclusion Approximately 2 years after GCO's launch, a third of MSM in the region are aware of the service with 1 in 10 GCO-aware men testing through the service. Given high intention to use GCO, these findings highlight the importance of continuing promotion efforts to raise awareness of the service among MSM.

\section{P4.114 THE APPLICATION OF A THEORETICAL MODEL TO FACILITATOR AND BARRIERS TO CHLAMYDIA TESTING IN GENERAL PRACTICE: A SYSTEMATIC REVIEW}

${ }^{1}$ Lorraine K Mcdonagh, ${ }^{2}$ John Saunders, ${ }^{3}$ Jackie Cassell, 'Greta Rait. 'University College London, London, UK; ${ }^{2}$ Public Health England, London, UK; ${ }^{3}$ University of Brighton, Brighton, UK

\subsection{6/sextrans-2017-053264.609}

Introduction: Chlamydia is a key health concern, with high economic and social costs. There were over 200000 chlamydia diagnoses made in England in 2015. The burden of Chlamydia is greatest among young people where the highest prevalence rates are found. Annual testing for sexually active young people is recommended; however, many of those at risk do not receive testing. General practice is an ideal setting for testing for multiple reasons; yet, testing here remains low. One theoretical model which may provide insight into the underpinnings of chlamydia testing is the Capability, Opportunity, and Motivation Model of Behaviour (COM-B model). This model proposes that behaviour (getting/providing a chlamydia test) is the result of capability, opportunity, and motivation. The aim of this review is to identify barriers and facilitators to chlamydia testing for young people in general practice, and use the COM-B Model to explore the theoretical mechanisms of action among these factors.

Methods Seven databases were searched to identify peerreviewed qualitative, quantitative, and mixed methods studies published after 2000. Data regarding study design and key findings were extracted. Data were analysed using thematic analysis and resultant factors were mapped onto the COM-B Model.

Results 315 papers were identified and screened; 28 were included for review. Results indicate that testing can be attributed to facilitators/barriers at the patient level (e.g., knowledge), provider level (e.g., time constraints), and system level (e.g., practice nurses). Regarding the COM-B Model, results suggest that knowledge of testing can be classified within the capability component; social stigma can be classified within the opportunity component; and personal beliefs about testing can be classified within the motivation component.

Conclusion The findings have relevance to healthcare professionals, policy-makers and commissioners in informing how best to improve the sexual health of young people.

\section{P4.115 HIGH UPTAKE OF EFFECTIVE EXPEDITED PARTNER THERAPY AMONG YOUNG WOMEN WITH STI AND THEIR PARTNERS IN SOUTH AFRICA}

${ }^{1}$ Nigel Garrett, ${ }^{1}$ Bhavna Maharaj, ${ }^{1}$ Farzana Osman, ${ }^{1}$ Nontobeko Ngubane, ${ }^{1}$ Hlengiwe Shozi, ${ }^{2}$ Noluthando Ngomane, ${ }^{2}$ Hope Ngobese, ${ }^{3}$ Andrew Gibbs, ${ }^{1}$ Ayesha Kharsany, ${ }^{4}$ Anne Rompalo, ${ }^{1}$ Adrian Mindel. 'Centre For The AIDS Programme of Research in South Africa (CAPRISA), Durban, South African Republic; ${ }^{2}$ Prince Cyril Zulu Clinic, Durban Municipality, Durban, South African Republic; ${ }^{3}$ South African Medical Research Council, Durban, South African Republic; ${ }^{4}$ Johns Hopkins University, Baltimore, USA

\subsection{6/sextrans-2017-053264.610}

Introduction Expedited Partner Therapy (EPT) for STIs delivered by the index case or through pharmacies has been implemented in some settings in the US. In South Africa, partner notification through the provision of a contact card to the patient reminding the partner to seek treatment has been unsuccessful (partner treatment rates of 17\%). Here, we explored the feasibility and acceptability of index case delivered EPT among young women in a high HIV incidence setting.

Methods HIV negative women, aged 18-40 years were screened for chlamydia, gonorrhoea (Xpert CT/NG) and trichomonas (OSOM) at an urban primary health care clinic. Women with STIs were treated with stat doses of antibiotics and were offered EPT packs, which included medication, condoms and an information leaflet for the current partner(s). An EPT questionnaire was administered telephonically one week later, and women were reviewed in clinic after 6 and 12 weeks.

Results: A total of 267 women, median age 23 (IQR 21-27), were screened and $63(23.6 \%)$ were diagnosed with a STI. Of these, 62/63 (98.4\%) were offered and 54/62 (87.1\%) accepted EPT for their regular partner. Two women chose EPT for one additional casual partner. At telephonic follow-up $47 / 54(87.0 \%)$ stated that they had successfully delivered EPT, i.e. the partner ingested the medication either observed $41 / 54$ $(75.9 \%)$ or unobserved $6 / 54(11.1 \%)$. Only five women (9.2\%) still had to deliver EPT and one partner refused. Some women reported that they $(17.5 \%)$ or their partners $(4.8 \%)$ experienced minor drug side effects consistent with antibiotic profiles, but no allergic reactions or social harms were reported. Of the first 53 women completing follow up reinfection rates were lower amongst women receiving EPT (1/47, $2.1 \%)$ compared to those not receiving EPT $(2 / 6,33.3 \%)$, $\mathrm{p}=0.031$.

Conclusion EPT uptake among young South African women and their partners was high and could play an important role in reducing reinfection rates and HIV risk. Larger studies should evaluate the feasibility of implementing this strategy at population level. 